\title{
AUTOMATED PLANNING, EXECUTION AND EVALUATION OF SIMULATION EXPERIMENTS OF SEMICONDUCTOR AMHS
}

\author{
Thomas Wagner \\ Clemens Schwenke \\ Klaus Kabitzsch \\ Technische Universität Dresden \\ Noethnitzer Strasse 46 \\ D-01187 Dresden, GERMANY
}

\author{
Germar Schneider \\ Infineon Technologies Dresden GmbH \\ Königsbrücker Strasse 180 \\ D-01099 Dresden, GERMANY
}

\begin{abstract}
Increasing variety and complexity of products in existing semiconductor factories cause an increased amount of production steps. Accordingly, this leads to a significant increase of non value added transportation processes. Therefore, transport and storage durations shall be minimized by optimal alteration of the given automated material handling system (AMHS). This can be achieved by simulation and analysis of possible AMHS alterations. However, this is a difficult task because of the system's complexity, the large amount of data and the high effort of manually modifying and testing many different AMHS alterations. In order to assist the system experts in executing these tasks, the authors suggest a method for automatic planning, execution and comparison of simulation experiments, including the automatic alteration of the transportation system's layout by introducing additional AMHS segments as shortcuts. The approach is feasible for existing simulation models as well as for generating simulations from the factory's core data.
\end{abstract}

\section{INTRODUCTION}

Nowadays, the most important challenge that existing semiconductor fabrication facilities (fabs) face, is ongoing change. Change of product mixes, increase of production volume as well as more complex integrated circuits contribute to the need to adapt existing fabs to new demands. Consequently, the manufacturing processes and layout of production equipment have to be changed as well as the corresponding automated material handling system as it has great impact on the overall fab performance as stated in (Kuhl and Christopher 2004).

But in order to stay competitive compared with newly planned and built facilities, the existing facilities have to make smart use of the existing tools, their locations and the connecting AMHS, so that for minimal costs of change a maximum of improvement effect can be expected. Therefore, facility layout design approaches with a maximum degree of freedom are not applicable. In contrast, this paper focuses on adapting existing transportation systems to new demands in a most effective way.

Facility design is interrelated with facility control. For example, facility layout examines spine layouts versus mesh layouts as well as best adaption to the shop floor principle, which is not in scope of this paper. Facility control addresses questions concerning dispatching and scheduling of wafer processing jobs on the so called fab level. On the underlying transportation level questions of operation of the AMHS have to be answered. The scope of this paper is on adaption of AMHS to increased production volume during ramp up, by the help of comprehensive but structured simulation experiments. As an example, an overhead vehicle based transport system of a real fab is examined.

In order to decrease overall travel times and cycle times, many approaches increase the amount of vehicles, e.g., (Govind, Roeder, and Schruben 2011). Another practical and inexpensive solution is to 
implement "shortcuts". A shortcut in the sense of this paper is an additional section of AMHS track components built into the existing transport system for the purpose of providing routing alternatives. This possibly decreases the transportation time of some transports by providing a shorter path between the source and destination locations. Because many tracks in the given transport system are unidirectional, they frequently cause the vehicles to perform detours if pickup destinations happen to be located shortly after delivery destinations. A well placed shortcut will shorten most routes and the corresponding rerouted traffic will block and delay very few original transports.

Unfortunately, there are no quick answers to the questions where and how to add shortcuts if an optimal quantity of many different routes shall be shortened. To the knowledge of the authors, no scientific approach that provides a method for systematic planning and examination of applicable alterations in given layouts without changing the existing routing algorithm is readily available at the moment. Therefore, the authors introduce a systematic approach for defining and testing applicable positions for shortcuts by simulating them. In addition, the automated adaption of weighting factors of the given shortest path routing algorithm is examined. This approach will later be added as a software module to a comprehensive framework for model building, analysing and simulating of transportation systems (Wagner, Schwenke, and Kabitzsch 2012).

The rest of the paper is structured as follows. Section 2 gives an overview of related work. In Section 3 the approach for structuring and automating the simulation models is described. An exemplary use case is depicted in Section 4. Finally, results are discussed in Section 5 so that conclusions are drawn and an outlook is provided.

\section{RELATED WORK}

In this paper, the authors introduce a work flow including automatic state model building and generating of simulation models. As a result, multiple simulation experiments can be executed automatically in order to test layout alterations in a structured manner. Therefore, the considered related work covers three directions, automatic building of state-transition models, facility design in semiconductor industry and generating of simulation models.

\subsection{State model building}

The authors implemented methods for automatic state model building previously and applied variations of them to different use cases before (Wagner, Schwenke, and Kabitzsch 2012), (Schwenke et al. 2012). In order to automatically construct a state-transition model event logs are required as input data. The resulting model can be interpreted as a highly aggregated information about observed state changes in a system. Therefore, the prerequisite is, that recorded events can be understood as notifications of state changes of entities.

In brief, the main essence of state model building is the extraction of events of a log file and aggregating them as a graph that consists of nodes and edges, representing states and transitions, respectively. In the case of material flow systems, events are recorded when wafer carriers enter tools, stockers or vehicles, where they are processed, stored or transported to a succeeding tool or stocker. Therefore, states represent wafer carriers being transported along transport system segments in a vehicle, being stored in a stocker or being processed in a tool. Transitions represent the change from one state to another.

The authors work on state model building was inspired by previous important work in this field. The use of discrete state models describing a system's behavior as a sequence of possible steps was done successfully before. For example, Kemper and Tepper (2005) extracted event discrete models from simulation trace file to identify partial deadlocks as well as queuing and blocking situations.

Moreover, state models are useful to monitor or identify business processes (Agrawal, Gunopulos, and Leymann 1998). van der Aalst et al. (2007) used state model building as a method for analyzing 
business processes, where events are generated when certain work steps begin and end. In so called process mining, process models shall be recovered or checked. Additionally, the relevance of business steps can be evaluated and performance indicators are calculated based on event logs.

In contrast to recovered business processes where humans often cause non-deterministic behavior, extracted models of AMHS are more deterministic but contain many more states so that automated tailored analysis approaches are needed.

The author's implementation of the state model building method allows to input a set of event data, that may be received from simulations or real fabs. The software tool extracts extended state-transition models for further analysis using a wide variety of performance indicators.

\subsection{Facility design in semiconductor industry}

As mentioned, facility design includes two separate although interrelated research areas, i.e. layout design and solving planning problems (Heragu 2006). Frequently, research on layout design and planning simplifies or ignores the details of the transportation system. In this paper, the layout of the underlying transport system is in focus, so that the transport performance will be increased.

A survey of problems, solution techniques, and future challenges in scheduling semiconductor manufacturing operations was published by Mönch et al. (2011). As they concluded, in the future AMHS have to be taken into consideration more precisely so that more precise planning solutions can be found.

In the following, exemplary research work is mentioned that is specialized on certain planning and layout design problems. The influence of available overhead hoist vehicles in a transport system on delivery times as well as load and unload times of wafers FOUPs was examined by Govind, Roeder, and Schruben (2011).

In contrast to optimizing the number of vehicles, Hsieh et al. (2012) proposed a segmented dual-track bidirectional loop design for an AMHS. The examined segmentation strategies result in reduced cycle times and increased stocker utilization regardless of the amount of used vehicles.

Hammel, Schmidt, and Schöps (2012) introduced an approach in order to reduce transport times by balancing the usage of routes by alteration of route weighting factors. The actual routing algorithm selects shortest paths based on these weighting factors. As a result, neither the given layout nor the actual routing algorithm needed to be changed.

Agrawal and Heragu provided a wide and thorough survey of AHMS in 300mm SC fabs (Agrawal and Heragu 2006). Many analyzed approaches are developed to design the layout of future transport systems, e.g., (Peters and Yang 1997). Some are developed to analyze and improve the operation of AMHS, e.g. (Nadoli and Pillai 1994). Because costs of developing and running detailed simulations are very high Agrawal and Heragu conclude that the matter calls for further investigation. Consequently, the authors of this paper developed a software framework that rapidly generates simulations so that changes in layouts can quickly be tested and compared to each other. Rapid to be generated and easy to be operated but rich in detail simulations are needed especially for testing improvements for existing fabs.

\subsection{Generating of simulations}

As Fowler and Rose (2004) stated, two of the grand challenges in simulation are still on-demand automatically built factory simulation models and user acceptance of simulations in industry. The latter can only be achieved by mastering the former. Accordingly, the automatic generation of simulation models has been subject to several research projects.

For example, several approaches for the simulation-based shop floor control had been introduced (Son, Wysk, and Jones 2003) or for aiding the factory automation experts during production planning (Randell and Bolmsjö ). However, these approaches are often developed in order to provide simulations of high level manufacturing processes and to test scheduling. In fab simulations, transports are usually ignored or their durations are simply modeled as distribution functions (Scholl et al. 2011). 
Few approaches exist in the field of generating realistic simulation models for transport systems, either based on extracting the plant layout and system components from e.g. CAD files (Paprotny, Zhao, and Mackulak ) or on interchanging data by use of the Core Manufacturing Simulation Data (CMSD) format (Johansson et al. 2007). Linear conveyor systems have been modeled with simple control logics and minimal routing alternatives, e.g., in semiconductor manufacturing (El-Nashar and El-Kilany 2007) or automotive industry (Wurdig and Wacker 2008).

Because costs of developing and running these detailed simulations of transport systems are very high, many approaches avoid to develop high detail level simulations and prefer structured reuse (Mackulak, Lawrence, and Colvin 1998). In contrast to this, the authors model transportation systems as discrete event state-transition-models, as described in Section 2.1 (Wagner, Schwenke, and Kabitzsch 2012). These abstract models are independent from particular simulation software. That is, first the desired alterations, e.g. layout changes, are applied to these abstract models in terms of manipulations of nodes and transitions. Second, the models are then used to generate the ultimate simulations.

\section{DESCRIPTION OF THE APPROACH}

The main goal of the developed methodology is to automatically generate and execute simulations of the fab's altered AMHS. Along with that, the simulation runs need to be evaluated in order to decide if the alterations represent an improvement or not. But the first step before generating new simulations, is to automatically decide where in the layout alterations shall be placed.

The reason why many different simulations need to be produced is the following. In contrast to simply changing parameter sets, e.g., number of vehicles, vehicle speeds, pickup durations or delivery durations, and apply them to the same simulation, in the use case of this paper physical layout alterations shall be tested. Layout alterations imply changes of rails for the OHVs in the real fab and thus imply changes of routes in the simulation model resulting in adaption of destination points and routing decision points. Therefore, each change of routes has to implemented as one individual simulation model that can be executed and evaluated separately.

Consequently, the approach consists of five general steps. As a first step, a model of the production area has to be constructed. The software framework, previously introduced by the authors, provides methods for automatically constructing such models based on different types of input data. The maximum size of the production area is mostly limited by the capabilities and computation time of the underlying simulator software.

In a second step, based on this model a simulation is generated and executed in order to analyze and evaluate the performance of this representation of the real fab. Third, layout alterations of this model have to be defined in structured manner, before the corresponding simulations can be generated and executed individually in step four.

In step five, the output data of all simulation runs is analyzed, compared to the simulation outputs of the original fab and evaluated so that the best solution can be picked by the user for implementation in the real fab. In the following, these steps will be described in detail, see Figure 1.

\subsection{Model building}

The generation of simulation models is based on a state-transition model. In a state-transition model states represent rail segments and stop locations for OHVs such as load ports at tools or stockers. Transitions represent the connections between rail segments as well as between rail segments and load ports. The authors introduced methods for automatically constructing such state-transition models before (Wagner, Schwenke, and Kabitzsch 2012; Schwenke et al. 2012).

In brief, the resulting model consists of several sets of entities, the set of states $S=\left\{s_{1}, s_{2}, \ldots, s_{n}\right\}$, the set of transitions $T \subseteq S \times S$, the set of moving loads $L=\left\{l_{1}, l_{2}, \ldots l_{m}\right\}$ and the set of paths $P \subseteq S^{*}$. Loads represent wafer carriers travelling in vehicles. Paths are the connections that the loads travel on and 


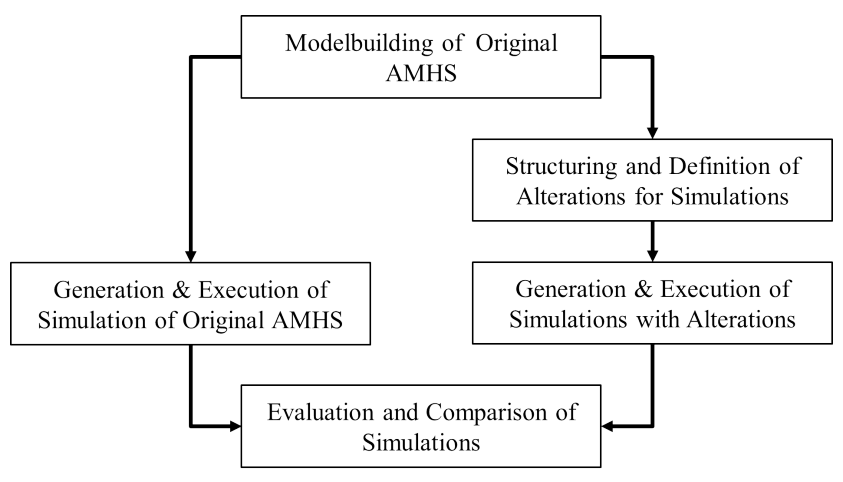

Figure 1: Workflow of simulation generating, execution and analysis.

visit states and are also called routes. If timestamped trace events have been recorded, the ordered set of events $E=\left\{e_{1}, e_{2}, \ldots, e_{N}\right\}$ with $e=(\tau \in Z, l \in L, s \in S)$ as an event and $Z=\left\{\tau_{1}, \tau_{2}, \ldots, \tau_{N}\right\}$ as the set of timestamps is part of the model and can be used for performance analysis. As mentioned, such models are called extended state-transition models, because they provide numerous time stamp based metrics for performance analysis.

The state-transition model can either be extracted from trace data of OHVs or wafer carriers. Alternatively, it can be composed from recorded transport job data and factory core data containing information about coordinates of tools and rails. Both methods have been implemented in a software framework by the authors. This software framework contains plugins, e.g., for deploying or replacing routing rules for routing decision points. In the work for this paper an additional plugin was developed for adding states and transitions that represent the segments of the to be tested newly added shortcuts.

\subsection{Generation and execution of simulation of the original AMHS}

In the second step, the simulation that represents the original transportation system has to be generated and executed. The movements of the vehicles as well as of the wafer carriers are recored in trace files. Precisely, at each state, e.g., equipment load port or routing alternative, a time stamp, wafer carrier ID, vehicle ID and station ID is recorded as an event and stored as a record in the trace file.

Subsequently, this trace file is used as input for the previously introduced software framework, so that an extended state-transition model, that provides performance indicators for each state, is derived. As mentioned, each state represents a rail segment or equipment where a time stamp of wafer carriers entering or leaving was recorded. Consequently, the performance indicators of each state can be calculated to evaluate and compare transport durations on particular routes or of the overall system. The set of these performance indicators is the baseline for all further comparisons, as described in Subsection 3.5.

If a rough estimation of the transport systems behaviour is sufficient, performance indicators can be extracted from recorded transportation event logs and used to parametrize the simulation, e.g., service times of equipment or rotary tables.

But in order to create most realistic simulation model out of the state-transition model, additional information about the single elements is required. Therefore, it is possible to assign a flexible set of additional properties to each of the basic model entities. Relevant attributes are for instance the element coordinates $\mathrm{x}$ and $\mathrm{y}$, lengths, vehicle movement speeds and vehicle capacities. In addition, data about the implemented routing strategies or boundary conditions of the material handling is required.

\subsection{Structuring and definition of alterations for simulations}

The most important aspect is the structured planning of the simulation experiments. Before simulation experiments can be carried out, it has to be decided where shortcuts should be placed. In order do this in 
a structured and comprehensive manner, in a first step a basic set of possible simulation experiments Exp, i.e. shortcut positions, is defined. This set exhibits all locations where shortcuts could be placed. But as explained later, not all positions will be actually useful.

The possible experiment positions can be derived from the given bay layout. For this purpose, the states in $S$ are extended with their starting and destination point locations as wells as size and shape attributes depending on the type of track element they are representing, e.g a straight segments, curve segment or destinations (load ports).

In the first step of the approach, all feasible positions are computed. At present, a shortcut can only be placed between two neighbouring, parallel track sections. This limitation is feasible because the currently investigated use cases do not allow more complex designs due to space limitations. The automation experts decided, only shortcuts between physically nearby AMHS sections are worth to investigate, see green, dashed lines in Figure 2. Consequently, all states representing linear track sections are searched and it is checked, whether their direct neighbours also represent linear elements of the same alignment with respect to the $x$ and $y$ positions of their starting and destination point locations. Possible shortcut positions are then defined along these segments, as long as they face each other, in a selectable interval. For each position found, a set of states $S^{\prime}$ is constructed, that consists of a number of states for each shortcut location. This set is then added to a set of experiments Exp. These states represent the 2 switches (curve sections), the linear track segment connecting them as well as references to the connected segments in the original AMHS layout $S_{C}$. A general example of possible shortcut positions is depicted in Figure 2.

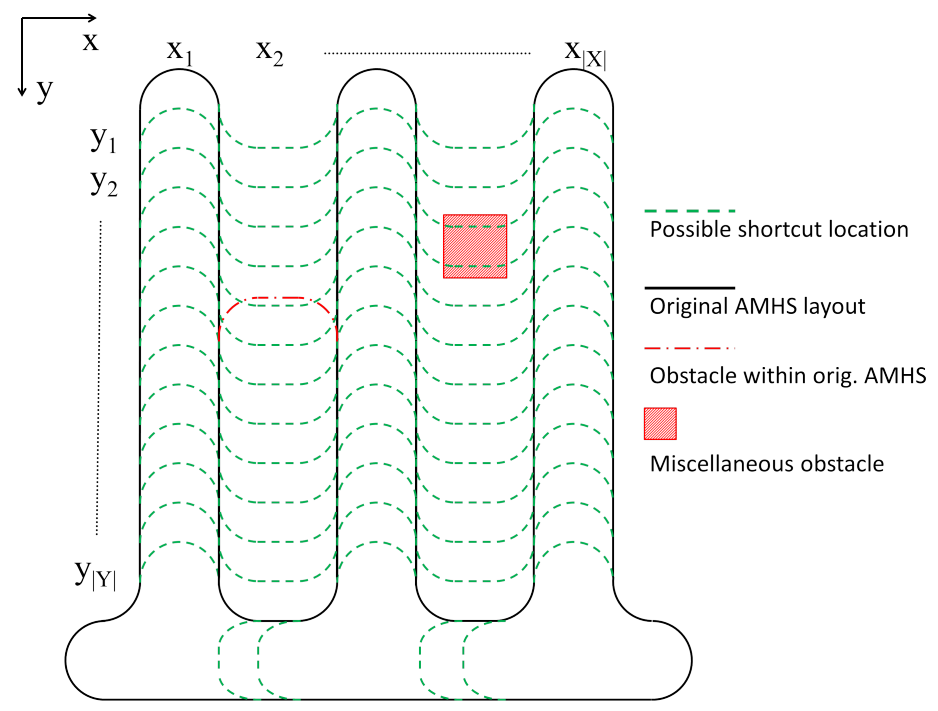

Figure 2: Exemplary grid of possible shortcut positions.

In a second step, shortcuts that would interfere with the existing AMHS components, i.e. elements in $S$, other than that they are connected to $\left(S_{C}\right)$ will be deleted. An example would be the shortcuts interfering with the slash dotted section in Figure 2. Such interferences are determined by checking for intersecting bounding boxes constructed around the shapes of each of the states in question.

Furthermore, additional constraints can be defined that restrict the possible amount of otherwise valid locations, i.e. positions within neighbouring parallel sections. In the use-case at hand, equipment load port positions must not be obstructed by the switches required when constructing the shortcuts. In the state-transition model, the deletion of not applicable shortcut positions is done by the definition of a set of states $S_{F}^{\prime}$ that represent such forbidden positions, e.g. load ports of tools or stockers. If one proposed shortcut element $s_{i j}^{\prime} \in S^{\prime}$ intersects with an element $s_{F}^{\prime} \in S_{F}^{\prime}$, the corresponding shortcut $S^{\prime}$ has to be deleted 
from the set or proposed states of experiments Exp. As a result a reduced set of applicable shortcuts, is derived.

In step three, the reduced set of possible shortcut locations is checked if shortcuts would cross the space where in reality miscellaneous objects are in the way. The information about these objects has to be extracted from auxiliary files, e.g., CAD-files that contain information about objects such as walls or gas and fluid pipes.

In the model this is done by the definition of a set of obstacle states $S_{O}^{\prime}$. The set $S_{O}^{\prime}$ is derived by parsing the areas that contain obstacles into states. If a state in a proposed shortcut $s_{i j}^{\prime} \in S^{\prime}$ intersects with one of the states in this forbidden set of states $S_{O}^{\prime}$ it has to be deleted from the set of experiments Exp.

In summary, the planning of shortcuts is carried out on three levels. First, a set of all shortcut positions is defined. Second, locations where connections to the given transport system are impossible are deleted from this set. Third, miscellaneous objects that represent obstacles have to be considered. Steps one to three are depicted as a program flowchart in Figure 3.

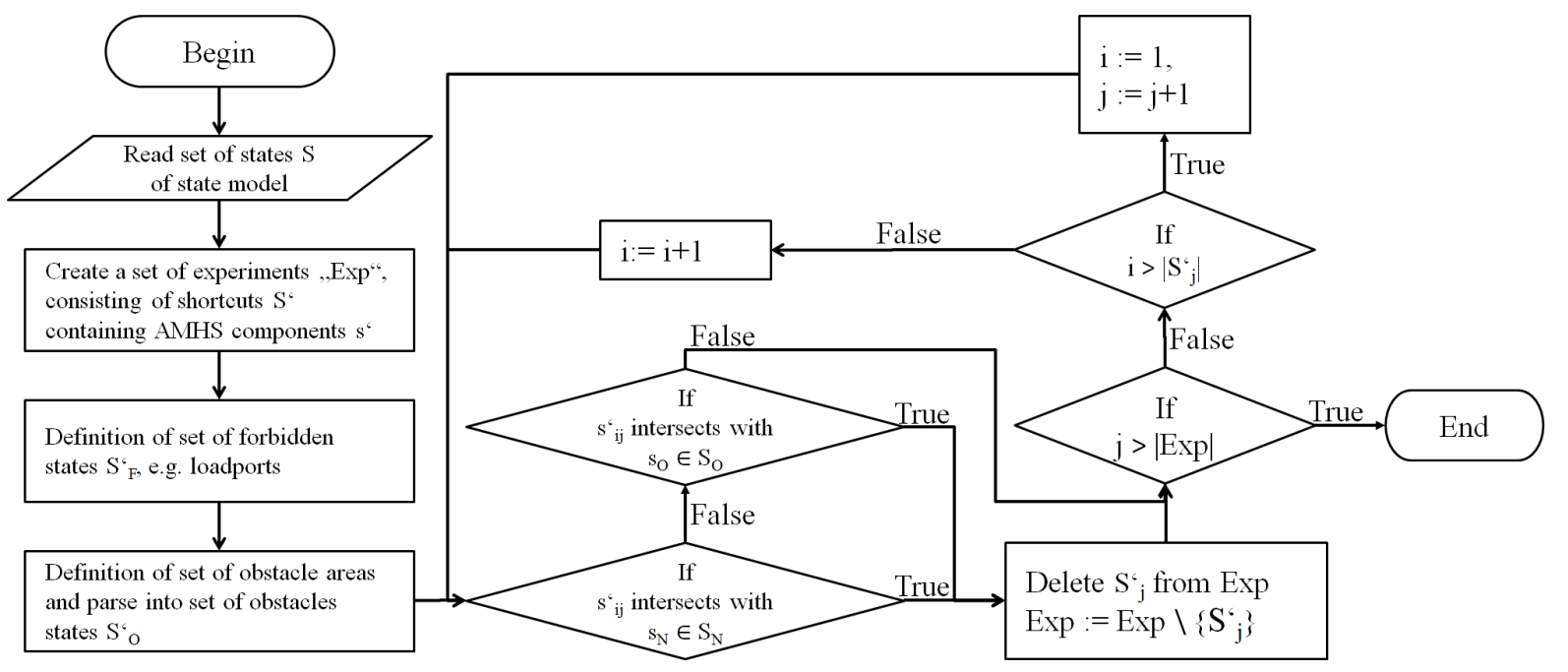

Figure 3: Program flowchart for the definition of shortcuts to be simulated.

\subsection{Generation and execution of simulations with alterations}

After the set of possible shortcuts is defined, the simulation models have to be generated and executed. The authors added a new software plugin to the previously introduced software framework, that allows the fully automatic generation, opening, execution, event logging and closing of the simulation models, that have been defined in Subsection 3.3.

In the use case of this paper, simulations are executed by the software AutoMod ${ }^{\complement}$, as it is common in semiconductor industry. But the plugin for operating AutoMod ${ }^{\complement}$ is interchangeable so that other simulation software could be used as well.

Along with the execution of the simulations, trace files are written so that analysis and comparing of solutions can be done as described in Subsection 3.5 and exhibited in Section 4.

\subsection{Evaluation of simulations}

After all simulation experiments have been executed and the corresponding trace files are stored, the evaluation has to be carried out. Two important exemplary performance indicators are the average travel time and the throughput of wafer carriers on the different paths between pick-up and delivery locations. Since the usage of transport times as an optimization criterion is simple, this section will focus on the 
maximum achievable throughput of wafer carriers per path. This is an important criterion because it reflects how different AMHS configurations can cope with upcoming ramp-up scenarios.

To measure the maximum achievable throughput for these relations, the simulation model must be tested with an increasing amount of transport jobs. These jobs are generated based on information about realistic product mixes and production routes from the fabs core data. An artificially higher amount of wafer starts per week is defined so that the AMHS simulation is tested with the maximum load it can handle. This maximum load is determined by automatically testing bigger and bigger loads until the storage queues of tools or stockers can no longer be emptied in a feasible time. Therefore, the queues always provide a lot to be transported and the vehicles are never idle. As a result, the maximum amount of performed jobs on each source destination relation defines the maximum throughput of the currently investigated AMHS configuration. For the purpose of AMHS layout comparisons, the large set of tested transport jobs is than kept the same for each experiment testing different locations for shortcuts.

The authors use the previously introduced software framework to automatically calculate the needed performance indicators. In the case of analysing throughputs, a matrix $D$ of path throughputs can be calculated.

The fields in the matrix contain the maximum achievable throughput $d_{i j}$ on a certain path $i$ with a new shortcut in a certain position $j$. There are $n$ routes representing connections between different pickup and delivery destinations and $m$ locations of tested shortcuts, see Formula 1 . The matrix $D$ can be used to evaluate the impact of one particular shortcut position to the throughput on the individual routes. Some shortcut positions cause an average improvement for nearly all routes. Other shortcut positions improve only a set of few routes but might impair other ones.

$$
D=\left(\begin{array}{lll}
d_{11} & d_{1 i} & d_{1 n} \\
d_{j 1} & d_{j i} & d_{j n} \\
d_{m 1} & d_{m i} & d_{m n}
\end{array}\right)
$$

In combination, the weighting factors for the routing algorithm, in AutoMod ${ }^{\complement}$ known as navigation factors, of the new shortcut are changed too. Thus, a new dimension for evaluating the results is introduced. As a result, for each tested weighting factor indexed by $w$, a new matrix $D_{w}$ must be calculated as shown in Table 2. That is, if $k$ weighting factors have to be tested, $k$ Matrices have to be computed. Thus, $d_{j i} \in D_{w}$ is the maximum achievable throughput on a certain path $i$ with a new shortcut in a certain position $j$ with a particular weighting factor indexed by $w$. The comparison of throughputs in combination with the weighting factors for an exemplary use case is described in detail in Section 4.

$$
D_{w}=\left(\begin{array}{lll}
d_{11} & d_{1 i} & d_{1 n} \\
d_{j 1} & d_{j i} & d_{j n} \\
d_{m 1} & d_{m i} & d_{m n}
\end{array}\right), w=1 \cdots k
$$

\section{EXEMPLARY USE CASE}

The investigated transportation system of a typical high volume $300 \mathrm{~mm}$ semiconductor fabrication plant is organized in many decoupled production bays served by overhead hoist transfer systems (intra-bay transport system) which are interconnected by a central overhead shuttle system (inter-bay transport system).

The approach described in the previous section has been successfully applied to the intra-bay systems. An exemplary step by step explanation of the application will now be presented using the transport system of a production bay who's model is depicted in Figure 4(a).

As described in Section 3, the second step of the approach after state model building is the generation and execution of the simulation of the unmodified system (baseline). This simulation provides the maximum throughput values $d_{i}$ for each possible path $i$ between sources and targets as a reference for comparison 


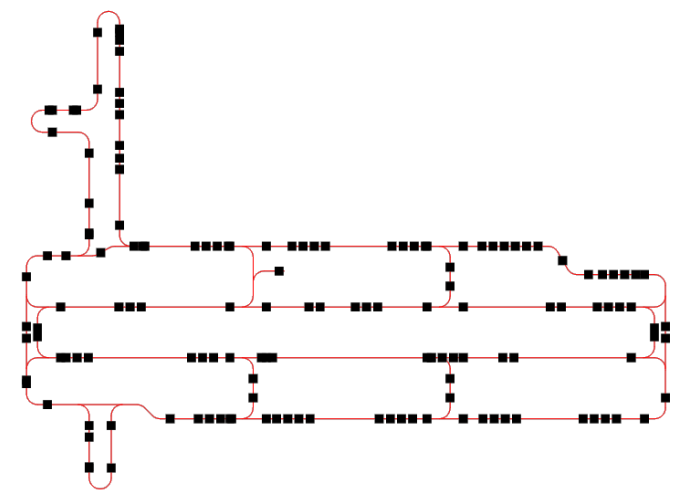

(a) Model of the Exemplary production bay.

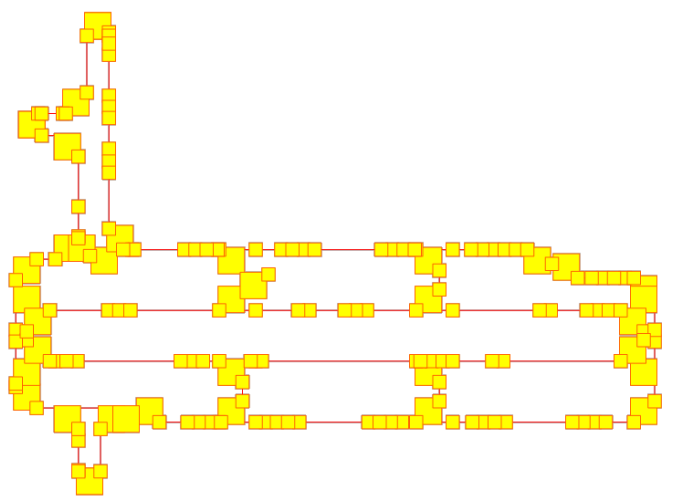

(b) Illegal shortcut positions (e.g. equipments or interfering transport system sections) highlighted using bounding boxes.

Figure 4: Model of the exemplary production bay, original and with obstacles highlighted.

with the results of the simulation experiments that will be automatically executed in step 4 . Table 1 shows the maximum throughput values of the baseline model for each route between stockers and tools.

Table 1: Transport count of the unmodified model for each Stocker-Equipment relation in a ten hour interval.

\begin{tabular}{rrrrrrrr}
\hline & \multicolumn{8}{c}{ Target Tool } \\
\hline Source Stocker & $101-104$ & $105-108$ & $109-112$ & $113-116$ & $117-120$ & $121-124$ & SUM \\
1 & 72 & 83 & 108 & 96 & 63 & 72 & $\mathbf{4 9 4}$ \\
2 & 70 & 68 & 103 & 102 & 71 & 66 & $\mathbf{4 8 0}$ \\
3 & 76 & 83 & 130 & 109 & 91 & 77 & $\mathbf{5 6 6}$ \\
SUM & $\mathbf{2 1 8}$ & $\mathbf{2 3 4}$ & $\mathbf{3 4 1}$ & $\mathbf{3 0 7}$ & $\mathbf{2 2 5}$ & $\mathbf{2 1 5}$ & $\mathbf{1 5 4 0}$ \\
\hline
\end{tabular}

After the acquisition of the reference throughput values, the set of experiments that have to be executed is planned automatically. Depending on the users requirements, multiple parameters can be tuned in each of the experiments. In this use case, a single shortcut should be placed in a location where the most throughput gains can be achieved. In addition, the weighting factor of the new shortcut is modified in several steps for each location. The importance of optimizing the weighting factors in OHT based AMHS was already shown by Hammel, Schmidt, and Schöps (2012) where it was used to balance the usage of the transport system, thus significantly increasing the throughput without worsening the delivery times.

The weighting factor is used by the routing algorithm to calculate the shortest route for the OHT vehicles from their current position to the target. The higher this value, the more a vehicle will avoid the new route depending on the length of the overall path. On the one hand, a large weighting factor may reduce the traffic density in this area of the transport system and thereby reduces the slowdown of the transit traffic of vehicles travelling to different locations. On the other hand, a large weighting factor may prevent the utilization of the shortcut for transports that may actually benefit from using it. Therefore, this parameter must be tuned for optimal performance. In this use case, the weighting factor is modified using a fixed range of values. For a more restrictive preselection of values, heuristic approaches, e.g., following the one described in Hammel, Schmidt, and Schöps (2012) can be used.

The possible shortcut locations are automatically constrained according to the method described in Section 3. 
In the first step, the shortcuts, consisting of two arcs with selectable angles and a straight rail segment, are placed according to the method proposed in the previous section. Afterwards, the sets of forbidden states within the AMHS $S_{F}^{\prime}$, e.g. tool loadports, and the set of obstacles $S_{O}^{\prime}$, e.g., walls or pipes, is parsed out of the fabs core data. A bounding box is constructed for each shortcut element and it is checked whether one of these boxes intersect with the states in $S_{F}^{\prime}$ or $S_{O}^{\prime}$. If this the case, the shortcut in question is deemed illegal and no experiment will be conducted using this location.

Once all of the valid positions are determined, simulation experiments are automatically conducted for every shortcut. For this purpose, the state-transition model of the unmodified production bay is extended by the states representing the shortcut's transport segments. Then the simulation model is generated and the new shortcut is parameterized using a weighting factor out of a selectable range of values. Figure 5(a) shows the simulation model of the exemplary production bay, extended by all valid shortcut locations for illustration purposes.

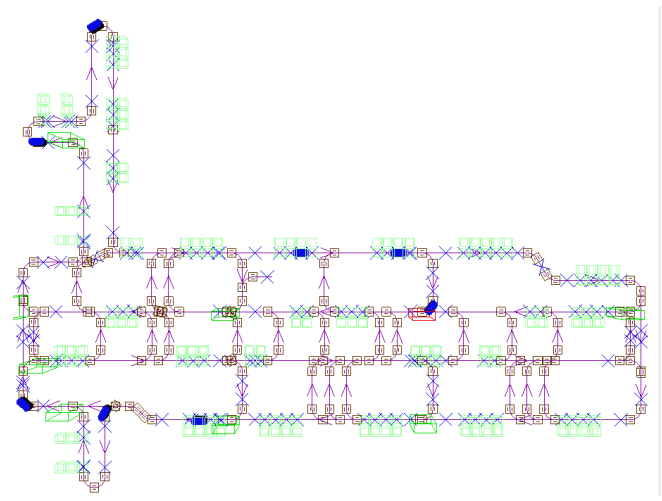

(a) The simulation model of the exemplary production bay, extended by all valid shortcut locations for illustration purposes.

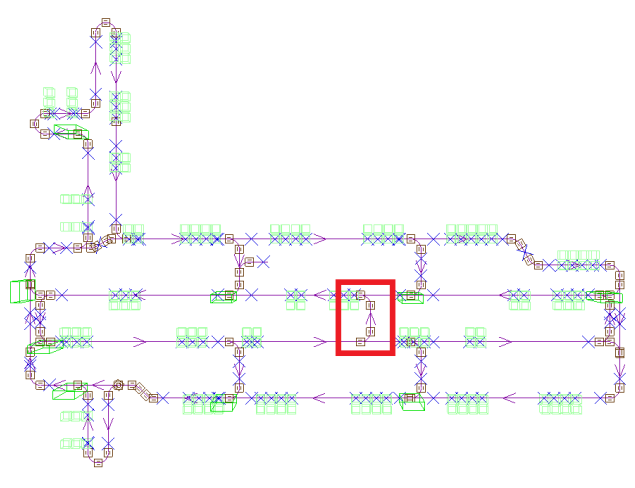

(b) The near optimal shortcut location (highlighted by the red rectangle) found during the simulation experiments.

Figure 5: Simulation model of the exemplary production bay, all valid shortcut locations and the near optimal solution

For the use case presented, 21 valid shortcut locations have been identified. Together with the selected range of weighting factors, ranging from 1 to 100 in steps of ten, 231 simulation experiments had to be executed. The input data, i.e. transport jobs, have been created as described in Section 3.5 and kept the same for each simulation run to ensure their comparability. Transient effects, like vehicle failures, have not been simulated yet since their occurrence is only an issue in long term simulation runs which have not yet been conducted during the feasibility study. In each experiment, transports in a 10 hour interval are simulated. In contrast, a typical transport job takes about one to three minutes. As a result, about 1600 transport jobs are carried out where inter arrival times of jobs vary. This way it is assured that good results do not occur by coincidence.

Each shortcut position and weighting factor is tested in a separate simulation model. As a result, the maximum throughputs can be compared and the simulation model that contains the shortcut in the best position can be determined.

On a Quad-Core Intel Core i7 CPU with 8 GB memory, 4 experiments could be executed in parallel, leading to a total execution time of around one hour. The resulting cumulated maximum throughput, i.e. the sum of the achieved transports of each individual path, for each experiment is shown in Figure 6, measured in achievable transports per 10 hours.

In the use case at hand, the best shortcut location, i.e. the one promising the biggest throughput gains, would be located at the centre of the system, using a weighting factor of 40 as depicted in Figure 5(b). 


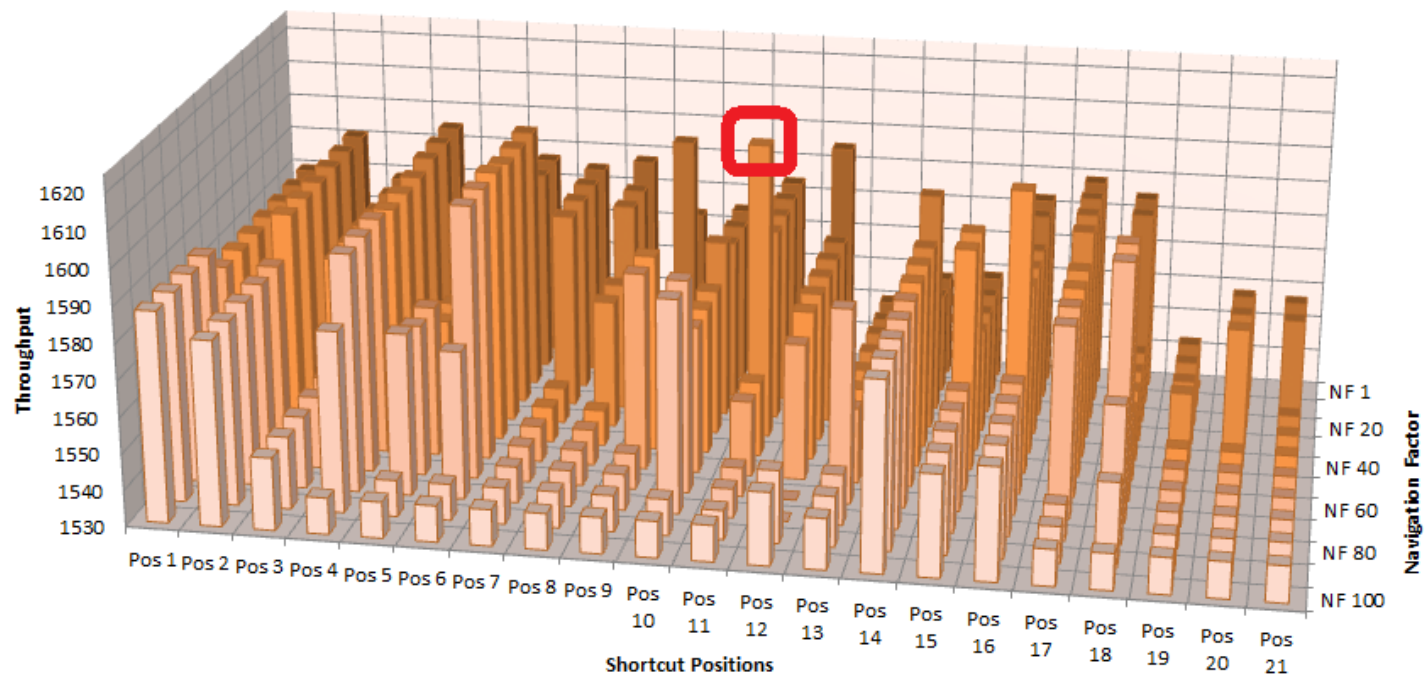

Figure 6: Cumulated throughput chart for all conducted experiments, the experiment exhibiting the largest throughput gain is highlighted in red.

With this configuration, the throughput enhancements shown in Table 2 can be achieved.

Table 2: Largest throughput gains achieved during the simulation runs for each Stocker-Equipment relation, measured in additional transports in a ten hour interval.

\begin{tabular}{rrrrrrrr}
\hline & \multicolumn{7}{c}{ Target Tool } \\
\hline Source Stocker & $101-104$ & $105-108$ & $109-112$ & $113-116$ & $117-120$ & $121-124$ & SUM \\
1 & 1 & 3 & 3 & 2 & 3 & 1 & $\mathbf{1 3}$ \\
2 & 4 & 3 & 7 & 7 & 2 & 5 & $\mathbf{2 8}$ \\
3 & 3 & 6 & 4 & 6 & 5 & 8 & $\mathbf{3 2}$ \\
SUM & $\mathbf{8}$ & $\mathbf{1 2}$ & $\mathbf{1 4}$ & $\mathbf{1 5}$ & $\mathbf{1 0}$ & $\mathbf{1 4}$ & $\mathbf{7 3}$ \\
\hline
\end{tabular}

\section{CONCLUSION AND OUTLOOK}

The manual planning of AMHS layout additions and changes is a tedious and time consuming process, even if simulation models of the current transport system state are available. The presented approach eases this process by providing the factory automation experts with automated simulation experiment planning and execution.

To ensure the applicability of the approach to a wide variety of transport systems and to enable the reuse of the analysis methods published before, an approved framework based on the abstract representation of transport systems as state-transition models was used as the basis of the approach. Generating the simulation from the mentioned state-transition model requires access to a significant amount of fab core data and recorded transport events, which may not be available. Instead, the method can also be applied to given simulation models if available. In that case, step one of the approach does not have to be carried out.

The results suggest that significant benefits can be achieved with relatively small changes to the AMHS such as placing a single shortcut and modifying its weighting factor. After having shown the feasibility of automatic simulation experiment planning and execution, further research will focus on creating modules 
to support the alteration of other aspects of the transport system in combination. This not only includes changes on the layout but also involves creating modules to alter other parameters of the simulation model like vehicle count, parking positions, equipment locations, routing strategies, implementation costs of the calculated solutions and so forth. In addition, the approach will be enhanced to take multiple, user definable production mixes and load scenarios into account while testing possible AMHS alterations.

However, this will result in a significant increase of simulation runs required to achieve near optimum solutions, thus sophisticated methods are needed to reduce the amount of simulation experiments as well as their runtime. As an example for the latter, meta modeling and estimation methods as described in Batarseh, Nazzal, and Wang (2010) will be evaluated.

In addition, fab simulation models as provided by Scholl et al. (2011) could be coupled with altered AMHS simulation models in order to comprehensively test the consequences on the overall fab performance.

\section{ACKNOWLEDGEMENTS}

This work originates in the context of the research and development project EPT300 (Grant Nr. 16N12182) funded by the German Federal Ministry of Education and Research (BMBF) and the ENIAC Joint Undertaking (JU).

\section{REFERENCES}

Agrawal, G., and S. Heragu. 2006. "A survey of automated material handling systems in 300-mm SemiconductorFabs". Semiconductor Manufacturing, IEEE Transactions on 19 (1): 112-120.

Agrawal, R., D. Gunopulos, and F. Leymann. 1998. "Mining process models from workflow logs". In Advances in Database Technology EDBT'98, edited by H.-J. Schek, G. Alonso, F. Saltor, and I. Ramos, Volume 1377 of Lecture Notes in Computer Science, 467-483. Springer Berlin Heidelberg.

Batarseh, O., D. Nazzal, and Y. Wang. 2010. "An Interval-Based Metamodeling Approach to Simulate Material Handling in Semiconductor Wafer Fabs". Semiconductor Manufacturing, IEEE Transactions on 23 (4): 527-537.

El-Nashar, A., and K. El-Kilany. 2007. "Reusable tool for 300mm intrabay AMHS modeling and simulation". In Proceedings of the 2007 Winter Simulation Conference, 1789-1797. Piscataway, New Jersey: Institute of Electrical and Electronics Engineers, Inc.

Fowler, J. W., and O. Rose. 2004. "Grand challenges in modeling and simulation of complex manufacturing systems". Simulation 80 (9): 469-476.

Govind, N., T. Roeder, and L. Schruben. 2011. "A Simulation-Based Closed Queueing Network Approximation of Semiconductor Automated Material Handling Systems". Semiconductor Manufacturing, IEEE Transactions on 24 (1): 5-13.

Hammel, C., T. Schmidt, and M. Schöps. 2012. "Network optimization prior to dynamic simulation of AMHS”. In Proceedings of the 2012 Winter Simulation Conference, WSC'12, 172:1-172:11. Piscataway, New Jersey: Institute of Electrical and Electronics Engineers, Inc.

Heragu, S. S. 2006. Facilities design. Iuniverse Inc.

Hsieh, C.-H., C. Cho, T. Yang, and T.-J. Chang. 2012. "Simulation study for a proposed segmented automated material handling system design for 300-mm semiconductor fabs". Simulation Modelling Practice and Theory 29:18-31.

Johansson, M., B. Johansson, A. Skoogh, S. Leong, F. Riddick, Y. T. Lee, G. Shao, and P. Klingstam. 2007. "A test implementation of the core manufacturing simulation data specification". In Proceedings of the 2007 Winter Simulation Conference, WSC '07, 1673-1681. Piscataway, New Jersey: Institute of Electrical and Electronics Engineers, Inc.

Kemper, P., and C. Tepper. 2005. "Trace based analysis of process interaction models". In Proceedings of the 2005 Winter Simulation Conference, 10 pp.-. Piscataway, New Jersey: Institute of Electrical and Electronics Engineers, Inc. 
Kuhl, M. E., and J. Christopher. 2004. "Capacity analysis of automated material handling systems in semiconductor fabs". In Proceedings of the 2004 Winter Simulation Conference. Piscataway, New Jersey: Institute of Electrical and Electronics Engineers, Inc.

Mackulak, G., F. Lawrence, and T. Colvin. 1998. "Effective simulation model reuse: a case study for AMHS modeling". In Proceedings of the 1998 Winter Simulation Conference, Volume 2. Piscataway, New Jersey: Institute of Electrical and Electronics Engineers, Inc.

Mönch, L., J. Fowler, S. Dauzre-Prs, S. Mason, and O. Rose. 2011. "A survey of problems, solution techniques, and future challenges in scheduling semiconductor manufacturing operations". Journal of Scheduling 14:583-599.

Nadoli, G., and D. Pillai. 1994. "Simulation in automated material handling systems design for semiconductor manufacturing”. In Proceedings of the 1994 Winter Simulation Conference, WSC '94, 892-899. Piscataway, New Jersey: Institute of Electrical and Electronics Engineers, Inc.

Paprotny, I., W. Zhao, and G. Mackulak. "Reducing model creation cycle time by automated conversion of a CAD AHMS layout design”. In Proceedings of the 1999 Winter Simulation Conference. Piscataway, New Jersey: Institute of Electrical and Electronics Engineers, Inc.

Peters, B., and T. Yang. 1997. "Integrated facility layout and material handling system design in semiconductor fabrication facilities". Semiconductor Manufacturing, IEEE Transactions on 10 (3): 360-369.

Randell, L. G., and G. S. Bolmsjö. "Database driven factory simulation: a proof-of-concept demonstrator". In Proceedings of the 2001 Winter Simulation Conference. Piscataway, New Jersey: Institute of Electrical and Electronics Engineers, Inc.

Scholl, W., B.-P. Gan, P. Lendermann, D. Noack, O. Rose, P. Preuss, and F. Pappert. 2011. "Implementation of a simulation-based short-term lot arrival forecast in a mature $200 \mathrm{~mm}$ semiconductor FAB". In Proceedings of the 2011 Winter Simulation Conference, 1927-1938. Piscataway, New Jersey: Institute of Electrical and Electronics Engineers, Inc.

Schwenke, C., T. Wagner, A. Gellrich, and K. Kabitzsch. 2012. "Event-based recognition and source identification of transient tailbacks in manufacturing plants". In Proceedings of the 2012 Winter Simulation Conference, 1-12. Piscataway, New Jersey: Institute of Electrical and Electronics Engineers, Inc.

Son, Y. J., R. A. Wysk, and A. T. Jones. 2003. "Simulation-based shop floor control: formal model, model generation and control interface". IIE Transactions 35 (1): 29-48.

van der Aalst, W., H. Reijers, A. Weijters, B. van Dongen, A. A. de Medeiros, M. Song, and H. Verbeek. 2007. "Business process mining: An industrial application". Information Systems 32 (5): 713 - 732.

Wagner, T., C. Schwenke, and K. Kabitzsch. 2012. "Modeling and wafer defect analysis in semiconductor automated material handling systems". In Proceedings of the 2012 Winter Simulation Conference, 1-12. Piscataway, New Jersey: Institute of Electrical and Electronics Engineers, Inc.

Wurdig, T. J., and R. Wacker. 2008. "Generic Simulation for Conveyor Systems". In Advances in Simulation for Production and Logistics Applications, 11-20: Stuttgart, Fraunhofer IRB Verlag 2008.

\section{AUTHOR BIOGRAPHIES}

THOMAS WAGNER is a PhD student at Dresden University of Technology. He is a member of the scientific staff of Professor Klaus Kabitzsch at the Chair of Technical Information Systems. He received his M.S. degree in computer science from Dresden University of Technology. His research interests include modeling, analysis and optimization of logistic systems. His e-mail is thomas.wagner2@ tu-dresden.de

CLEMENS SCHWENKE is a PhD student at Dresden University of Technology. He is a member of the scientific staff of Professor Klaus Kabitzsch at the Chair of Technical Information Systems at the Department of Computer Science. He received his M.S. degree in Electrical Engineering from Dresden University of Technology. His research interests include automation as well as modeling, analysis and 
design of discrete event systems. His e-mail is clemens.schwenke@tu-dresden.de

GERMAR SCHNEIDER is a manager at Infineon Technologies Dresden GmbH for "Thin Wafer Handling" and project leader for automation projects within the Eniac funding projects EPT300 and EPPL. He received a Diploma and a Ph.D. in analytical chemistry and works since 18 years in different positions at Infineon Technologies. In the recent years he filled management positions in processing, manufacturing and maintenance departments and was head of an automation and factory integration group. His e-mail is germar.schneider@infineon.com

KLAUS KABITZSCH is the head of the department of technical computer sciences at the Dresden University of Technology. He received a Diploma and a Ph.D. in electrical Engineering and communications technology. His current projects have their focus on the automation domain, component based software design, design tools for networked automation, energy and quality management, data analysis, advanced process control and predictive technologies. He is a member of IEEE, VDE and GI. His e-mail is klaus.kabitzsch@tu-dresden.de 